JIOM Nepal. Volume 41 Number 3. December 2019, page 59-62.

\title{
Antimicrobial Susceptibility Pattern of Acinetobacter calcoaceticus-Acinetobacter baumannii Complex Isolated from Sputum in a Tertiary Care Hospital
}

\author{
Jyotshna Sapkota', Manisha Sharma ${ }^{1}$, Deepti Shrestha ${ }^{2}$, Beena Jha ${ }^{1}$ \\ 'Department of Microbiology, Kathmandu Medical College and Teaching Hospital, Sinamangal, Kathmandu, Nepal, \\ 2Department of Pharmacology, Nepal Medical College and Teaching Hospital, Jorpati, Kathmandu, Nepal
}

Corresponding author:

Jyotshna Sapkota, MBBS, MD

Department of Microbiology, Kathmandu Medical College and Teaching Hospital, Sinamangal, Kathmandu, Nepal Email:21jyots@gmail.com

Submitted : November 6, 2019

Accepted : December 4, 2019

\begin{abstract}
Introduction

Acinetobacter calcoaceticus-Acinetobacter baumanni (ACB) complex is one of the commonest cause of hospital acquired and ventilator associated pneumonia. Multidrug resistant Acinetobacter species have become a matter of huge concern. This study was done to find out the antibiotic susceptibility pattern of Acinetobacter calcoaceticus-Acinetobacter baumanii complex from sputum samples.

\section{Methods}

This descriptive cross-sectional study was carried out in Clinical Microbiology laboratory from July 2018 to Jan 2019 after ethical approval. Acinetobacter calcoaceticus-Acinetobacter baumannii complex was identified on the basis of its microscopy and morphological characteristics followed by biochemical tests. Antibiotic sensitivity test of isolated pathogens was done using Muller Hinton Agar by Kirby-Bauer method.

\section{Results}

Of the 384 culture positive sputum specimen, 76 (19.80\%) were Acinetobacter calcoaceticus-Acinetobacter baumannii complex. Most of the isolates were resistant to commonly used antibiotics, $72.36 \%$ of the isolates were multidrug resistance and $3.95 \%$ isolates were resistant to tigecycline.

\section{Conclusion}

This study provides valuable information regarding prevalence of Acinetobacter calcoaceticus-Acinetobacter baumannii complex from sputum specimen. The alarming number of Multidrug resistance isolates is worrisome finding. Antibiotics like Tigecycline and Colistin which is still sensitive to isolates should be cautiously used only in MDR cases.
\end{abstract}

Keywords: Acinetobacter, ACB complex, antibiotic resistance, MDR, Nepal

\section{INTRODUCTION}

A cinetobacter calcoacetius-Acinetobacter baumannii complex (ACB) organisms are aerobic, non-fermenting gram negative coccobacilli. Clinically ACB organisms mainly cause health-care associated infections but are also associated with community acquired infections. ${ }^{1}$ The respiratory tract is an important site of colonization and is the most frequent site of infection. ${ }^{2}$ The most common clinical condition associated with these microorganisms is hospital- acquired pneumonia (HAP), particularly for patients receiving mechanical ventilator assistance. ${ }^{3}$

Despite advances in health care and wide variety of antibiotics, life threatening infections caused by ACB complexes are considered as one of the major health problem. Emergence of infections caused by multidrug resistant (MDR) strains of ACB complex increase morbidity, mortality and impose an enormous burden on health care cost. Alarmingly some strains of ACB exhibites resistance to 'lastresort' drugs like tigecycline, Polymixin B and 
Colistin sulfate.The resistance pattern of bacteria changes over time and varies from place to place. Therefore regular surveillance is needed treat infections empirically and effectively. ${ }^{4,5}$

This study aimed to determine the prevalence of ACB complex in sputum sample, their antibiotic susceptibility pattern and frequency of MDR ACB complexes in our setting

\section{METHODS}

A hospital based descriptive cross sectional study was carried out in Clinical Microbiology Laboratory of Kathmandu Medical College and Teaching Hospital (KMCTH), Kathmandu Nepal from the month of July 2018 to Jan 2019. Ethical approval was received from Institutional Review Committee. All the sputum samples, appropriately collected, labelled, properly transported and processed for aerobic bacterial cultures. A total of 384 culture positive sputum isolates were included in this study. Samples received were processed according to standard microbiological procedures. ${ }^{6}$ Suspected colonies of ACB complex were further processed. Identification of ACB complex was done on the basis of colony character, gram's staining and biochemical tests. ${ }^{7}$ Antimicrobial susceptibility of all isolates was determined by the standard Kirby Bauer disk diffusion method according to norms of Clinical Laboratory Standards Institute (CSLI). Antibiotics included were Amikacin $(30 \mu g)$, Ceftriaxone (30 $\mu g)$, Ceftazidime $(30 \mu \mathrm{g})$, Ciprofloxacin $(5 \mu \mathrm{g})$, Pipracillin (30 $\mu \mathrm{g})$, Piperacillin/Tazobactum (100/10 $\mu \mathrm{g})$, Imipenem $(10 \mu \mathrm{g})$, Tigecycline $(15 \mu \mathrm{g})$, Colistin $(20 \mu \mathrm{g})$ and Polymyxin B (300units). ${ }^{8}$ In this study, if the isolates were resistant to at least three classes of first line antimicrobial agents, they were regarded as MDR. ${ }^{9}$

\section{RESULTS}

Out of 384 culture positive sputum sample a total of $76(19.80 \%)$ ACB organisms were isolated, out of which $40(52.7 \%)$ were from males and $36(47.4 \%)$ were from females. The mean age was 60.46 ranging from 16 to 98 years, with most growths from $61-80$ years of age $(44.74 \%)$. Prevalence of ACB complex among age group 6180 was found to be statistically highly significant ( $P$ value $<0.001$ ).

The antimicrobial susceptibility pattern of the ACB complex showed resistance to most of the antibiotics. Highest resistance was seen to Ceftriazone (86.84\%), Pipracillin (84.21\%) and Ciprofloxacin (81.58\%). Resistance to Ceftazidime was (80.7\%) and that of Amikacin
Table 1. Antimicrobial susceptibility pattern of $A C B$ complex

\begin{tabular}{lccc}
\hline Antibiotics & $\begin{array}{c}\text { Resistant } \\
\text { (\%) }\end{array}$ & $\begin{array}{c}\text { Intermediate } \\
\text { sensitive (\%) }\end{array}$ & $\begin{array}{c}\text { Sensitive } \\
\text { (\%) }\end{array}$ \\
\hline Amikacin & 77.63 & 2.63 & 19.74 \\
Ceftriazone & 86.84 & 2.63 & 13.16 \\
Ceftazidime & 80.70 & - & 19.30 \\
Ciprofloxacin & 81.58 & - & 18.42 \\
Colistin & 0.00 & - & 100 \\
Meropenem & 61.84 & - & 35.53 \\
Pipracillin & 84.21 & - & 15.79 \\
Pipracillin/ & 73.68 & 1.32 & 25.00 \\
tazobactum & & & \\
Tigecycline & 3.95 & 1.32 & 94.74 \\
\hline
\end{tabular}

was $(77.63 \%)$. Highest sensitivity was seen to Colistin (100\%), Tigecycline (94.74\%). Over all sensitivity and resistancee pattern is shown in Table 2.

Out of 76 ACB complex, $72.36 \%$ were MDR.

\section{DISCUSSION}

The prevalence and resistance of ACB complex to antibiotics has amplified considerably over the past few years. In this study, the prevalence of ACB complex among sputum isolates is $19.80 \%$, which is similar to studies done by Nepal et al in 2016 which showed the prevalence of $17.33 \%$, but our result is higher than the study done in 2013 by Mishra et al which showed prevalence of $11.23 \%$ and Tamang et al in 2005 reported prevalence as $3.9 \%$ from respiratory secretions. These findings suggest an increasing trend in the prevalence of ACB complex. ${ }^{10-12}$

Though ACB complex is known to have low virulence but among gram negative bacteria they show resistance to majority of commonly used antibiotics. This study demonstrated higher prevalence of drug resistance among the ACB complex towards majority of the antibiotics. Our study reported high resistant to cephalosporins and Fluroquonolones. Ceftazidime resistance was seen in $80.7 \%$ which is similar to the studies done in Nepal by Amatya et al and Shrestha et al. ${ }^{13,14}$ This is probably due to the widespread use of thirdgeneration cephalosporins in our setup. Some of the studies done in Nepal around the world also showed $100 \%$ resistance to Ceftazidime. ${ }^{15,16}$ Ciprofloxacin resistance was $81.58 \%$ in our study which is higher than other studies conducted in Kathmandu which showed resistance of $64.5 \%$ and $68.7 \%{ }^{11,13}$ The differences in the sample types, size, source, site of study, antibiotics usage, and 
the hospital infection control practices followed can influence the resistance pattern. Equally likely is that the data reflects an actual increase in the resistance to these antibiotics over the years as there is wide use of antibiotics available over the counter without specific laboratory tests in our country.

Once considered as drug of choice for Acinetobacter infections, Carbapenem resistanct strains are reported worldwide. ${ }^{17,18}$ In our study Meropenem was resistant in $61.84 \%$. Study conducted by Zelleri et al suggested carbapenem resistance is higher in places where they are used extensively. ${ }^{19}$ Carbapenems are used extensively in Nepal which explains increase in resistance to these antibiotics.

The frequency of MDR ACB complex in our study was $72.36 \%$ which is comparable to Amatya et al in 2018. ${ }^{13}$ Other parts of Southeast Asia have also shown increasing MDR trend. ${ }^{20}$ This current finding implies that only higher antibiotics like Colistin and Tigecycline as a treatment option to combat MDR ACB complex.

Antibiotics like tigecycline and colistin should be judiciously used only in MDR cases. Our study did not document any resistance to Colistin which was similar to previous studies done in Nepal. ${ }^{11,13,14}$ But $3.95 \%$ of isolates were tigecycline resistant. Tigecycline is one of the "last-resort" antimicrobial agents for antibiotic resistance in ACB infection. Though it has been used for only about 10 years, significant percentage of resistance has been observed in many studies done in Nepal and elsewhere. $1,13,16$

\section{CONCLUSION}

The present study establishes ACB complex as important isolate from sputum sample. With increase in prevalence of MDR ACB complex cautious use of antibiotics should be practiced. Irrational use of antibiotics, absence of antimicrobial stewardship program in hospitals, lack of surveillance and reporting system, failure to observe infection control practices like hand washing and barrier nursing could be some reasons for this problem. Although no isolate exhibited resistance to Colistin and few isolates were Tigecycline resistant screening test and MIC determination are recommended in monitoring the response to therapy and for early detection of impeding resistance among local strains and these antibiotics should only be used in MDR cases.

\section{CONFLICT OF INTEREST}

None declared.

\section{REFERENCES}

1. Allen DM, Hartman BJ. Acinetobacter species. In: Mandell GL, Bennett JE, Dolin R, editors. Mandell, Douglas and Bennett's Principles and Practice of Infectious Disease. 6th ed. Philadelphia: Elsevier Churchill Livingstone; 2005. pp. 2626-32

2. Wong $D$, Nielsen $T B$, Bonomo RA, Pantapalangkoor $P$, Luna B and Spellberg B. Clinical and Pathophysiological Overview of Acinetobacter Infections: a Century of Challenges. Clin Microbiol Rev. 2017; 30:409-47

3. Yang $Y S$, Lee $Y T$, Huang $T W$, Sun JR, Kuo SC, Yang $\mathrm{CH}$. Acinetobacter baumannii nosocomial pneumonia: is the outcome more favorable in non-ventilated than ventilated patients? BMC Infect Dis. 2013;13:142.

4. Durante-Mangoni E, Zarrilli R. Global spread of drug-resistant Acinetobacter baumannii: molecular epidemiology and management of antimicrobial resistance. Future Microbiol. 2011;6(4):407-22.

5. Ko KS, Suh JY et al. High rates of resistance to colistin and polymyxin $B$ in subgroups of Acinetobacter baumannii isolates from Korea. J Antimicrob Chemother. 2007;60(5):1163-67

6. Isenberg HD. Clinical Microbiology Procedures handbook. 2nd edition. Washington DC: ASM press; 2004.

7. Weinstein MP, Patel JB, Bobenchik AM et al. Clinical and Laboratory Standards Institute. Performance standards for antimicrobial susceptibility testing; Twenty-second informational supplement. 2012:88-9.

8. CLSI (2018). Performance standards for anitmicrobial susceptibility testing, 28th informational supplement, vol.38. CLSI Document M100-S28. Wayne (PA), CLSI

9. Adams-Haduch JM, Paterson DL, Sidjabat $\mathrm{HE}_{\text {, }}$ et al. Genetic basis of multidrug resistance in Acinetobacter baumannii clinical isolates at a tertiary medical center in Pennsylvania. Antimicrob Agents Chemother 2008; 52:3837-43

10. Nepal R, Shrestha B, Joshi DM, Joshi RD, Shrestha S, Singh A. Antibiotic Susceptibility Pattern of Gram-negative Isolates of Lower Respiratory Tract Infection. J Nepal Health Res Counc 2018 Jan-Mar;13; 16(38): 22-6

11. Mishra SK, Rijal BP and Pokhrel BM. Emerging threat of multidrug resistant bugs-Acinetobacter calcoaceticus baumannii complex and Methicillin resistant Staphylococcus aureus. BMC research notes 2013; 6: 98

12. Tamang MD, Dey S, Makaju RK, Jha BK, Shivananda $P G$, Bhramadatan KN: Bacterial aetiology of lower respiratory tract infection in patients attending Manipal Teaching Hospital. J Nepal Assoc Med Lab Sci. 2005, 7: 15-19.

13. Amatya R, Acharya D. Prevalence of tigecycline resistant multidrug resistant Acinetobacter calcoaceticus- Acinetobacter baumannii complex 
from a tertiary care hospital in Nepal. Nepal Med Coll J. 2015; 17(1-2): 83-86

14. Shrestha M and Khanal B. Acinetobacter Species: Phenotypic Characterization and Antimicrobial Resistance. J Nobel Medical College 2013; 2: 438.

15. Boral B, Unaldi O, Ergin A, Durmaz R, Eser OK. A prospective multicenter study on the evaluation of antimicrobial resistance and molecular epidemiology of multidrug-resistant Acinetobacter baumannii infections in intensive care units with clinical and environmental features. Ann Clin Microbiol Antimicrob. 2019 Jul; 18(1): 19

16. Shrestha S, Tada T, Miyoshi-Akiyama $T$ et al. Molecular epidemiology of multidrug-resistant Acinetobacter baumannii isolates in a university hospital in Nepal reveals the emergence of a novel epidemic clonal lineage. Int J Antimicrob Agents 2015; 46:526-31

17. Fontana C, Favaro M, Minelli S et al. Acinetobacter baumannii in intensive care unit: A novel system to study clonal relationship among the isolates. BMC Infect Dis 2008; 8:79.

18. Manikal VM, Landman D, Saurina G, Oydna E, Lal $\mathrm{H}$ and Quale J. Endemic carbapenem -resistant Acinetobacter species in Brooklyn, New York: citywide prevalence, interinstitutional spread, and relation to antibiotic usage. Clin infect Dis 2000; 31: 101-6.

19. Zarrilli R, Giannouli M, Tomasone F, Triassi $\mathrm{M}$ and Tsakris A. Carbapenem resistance in Acinetobacter baumannii: the molecular epidemic features of an emerging problem in health care facilities. J Infect Develop Countr 2009; 3: 335-41

20. Teerawattanapong $N$, Panich $P$, Kulpokin $D$, et al. A systematic review of the burden of multidrugresistant healthcare-associated infections among intensive care unit patients in Southeast Asia: the rise of multidrug-resistant Acinetobacter baumannii. Infect Control Hosp Epidemiol. 2018;39(5):525-533 